Article

\title{
Determination of $2^{\prime}$-Fucosyllactose and Lacto-N-neotetraose in Infant Formula
}

\author{
Sean Austin ${ }^{1, * \mathbb{C}}$, Denis Cuany ${ }^{1}$, Julien Michaud ${ }^{1,2}$, Bernd Diehl ${ }^{3}$ and Begoña Casado ${ }^{1,4}$ \\ 1 Nestlé Research, Vers-Chez-Les-Blanc, 1000 Lausanne, Switzerland; denis.cuany@rdls.nestle.com (D.C.); \\ julien.x.michaud@gsk.com (J.M.); begona.casado@nestle.com (B.C.) \\ 2 Current address: GSK Consumer Healthcare S.A., Route de l'Etraz 2, 1260 Nyon, Switzerland \\ 3 Spectral Services, Emil-Hoffmann Strasse 33, D-50996 Köln, Germany; diehl@spectralservice.de \\ 4 Société des Produits Nestlé SA, Nutrition SBU, Rue d'Entre-deux-Villes 10, \\ 1814 La-Tour-De-Peilz, Switzerland \\ * Correspondence: sean.austin@rdls.nestle.com; Tel.: +41-21-785-8050
}

Received: 15 August 2018; Accepted: 10 October 2018; Published: 16 October 2018

\begin{abstract}
Human milk oligosaccharides (HMO) are the third most abundant solid component of human milk. It is likely that they are responsible for at least some of the benefits experienced by breast-fed infants. Until recently HMO were absent from infant formula, but $2^{\prime}$-fucosyllactose (2'-FL) and lacto-N-neoteraose (LNnT) have recently become available as ingredients. The development of formula containing these $\mathrm{HMO}$ and the quality control of such formula require suitable methods for the accurate determination of the HMO. We developed two different approaches for analysis of 2'-FL and $\mathrm{LNnT}$ in formula; high performance anion exchange chromatography with pulsed amperometric detection (HPAEC-PAD) and hydrophilic interaction liquid chromatography with fluorescence detection (HILIC-FLD). In lab trials using blank formula spiked with the two oligosaccharides, both approaches worked well with recoveries of 94-111\% (HPAEC-PAD) and 94-104\% (HILIC-FLD) and RSD (iR) of 2.1-7.9\% (HPAEC-PAD) and 2.0-7.4\% (HILIC-FLD). However, when applied to products produced in a pilot plant, the HPAEC-PAD approach sometimes delivered results below those expected from the addition rate of the ingredients. We hypothesize that the oligosaccharides interact with the formula matrix during the production process and, during sample preparation for HPAEC-PAD those interactions have not been broken. The conditions required for labeling the HMO for detection by the FLD apparently disrupt those interactions, and result in improved recoveries. It is likely that both analytical approaches are appropriate if a suitable extraction process is used to recover the HMO.
\end{abstract}

Keywords: human milk oligosaccharides; liquid chromatography; infant formula; 2'-FL; LNnT

\section{Introduction}

Human milk oligosaccharides (HMO) are the third most abundant solid component of human milk after lactose and fats. HMO are nondigestible oligosaccharides and, among other things, are believed to be implicated in protecting the infant from infections, and in the establishment of a healthy gut microbiota [1-5]. Although more than 150 different HMO have been identified [6], quantitative data only exists for about 30 of the HMO in milk [7].

The HMO can be roughly classified in to three different types; fucosylated HMO, sialylated $\mathrm{HMO}$, and the core HMO. This is not a rigid separation of structures, since certain HMO can be both fucosylated and sialylated and thus can be classified in to two of the groups. Within the fucosylated HMO, 2'-fucsyllactose (2'-FL) is typically the most abundant, and has been measured at levels up to $8 \mathrm{~g} / \mathrm{L}$ [8], but most typically is present in milk at levels between 2 and $3 \mathrm{~g} / \mathrm{L}$ [7]. The potential 
health benefits of fucosylated HMO have been studied and there is good evidence suggesting that they provide protection against diarrhea [9-11]. Lacto-N-neotetraose (LNnT) is one of the more abundant core structures, which can be found without further modification in human milk. It has been detected at levels between 0.010 and $4.1 \mathrm{~g} / \mathrm{L}$ [12,13], and is typically found at levels between 0.3 and $1.1 \mathrm{~g} / \mathrm{L}$ [7]. Levels of both LNnT and 2'-FL are quite dynamic, with large variations in concentrations observed between individuals, and depending on stage of lactation.

Despite their abundance in human milk, HMO have not historically been a component of infant formula, largely because their function was unclear and because they were difficult to obtain; they are not present in significant quantities in the milk of farmed animals and routes to produce them on an industrial scale have been prohibitively expensive. However, as science has progressed the potential roles of HMO have started to be elucidated [1,2]. Concomitantly, routes to synthesize HMO on a large scale and at reasonable cost have been developed [14]. The supply of HMO is now such that they can be introduced to infant formula [15-17], and 2016 saw the first launches of formula containing $2^{\prime}$-FL in the USA and 2'-FL and LNnT in Europe.

During the development of formula containing $\mathrm{HMO}$, methods had to be developed to determine $\mathrm{HMO}$ concentration in the finished product. Apart from being used for quality control purposes to ensure labeling compliance, the methods were also needed to confirm the levels of HMO in products destined for tolerance and safety trials, efficacy trials, and to ensure HMO stability during the formula production process. In this paper we describe two chromatographic methods for the determination of 2 -FL and LNnT in infant formula. One method is based on high performance anion exchange chromatography with pulsed amperometric detection (HPAEC-PAD) and the other on hydrophilic interaction chromatography with fluorescence detection (HILIC-FLD). Both techniques have previously been applied for the analysis of HMO in human milk [18,19]. Applied to spiked infant formula, both methods performed well. However, applied to some formula produced on pilot or industrial plants the HPAEC-PAD method returned lower results, while the HILIC-FLD method returned results more in line with expectations. We suspect the sample preparation procedure for the HILIC-FLD method disrupts interactions between $\mathrm{HMO}$ and the product matrix, those interactions are not disrupted using the more simple "dilute and shoot" approach used with HPAEC-PAD.

\section{Results}

In order to determine the quantity of oligosaccharides added to the formula by chromatography it was necessary to obtain good quality, quantitative, analytical standards. At the start of this study such standards were not readily available. The HMO ingredients themselves were therefore used as the standards. In order to establish the purity and moisture content of the ingredients, quantitative nuclear magnetic resonance spectroscopy (qNMR) was employed. Using the well-characterized material it was possible to develop chromatographic methods for the analysis and validate them.

\subsection{Quantitative Nuclear Magnetic Resonance Spectroscopy (qNMR)}

Several batches of oligosaccharide ingredients were analyzed by qNMR to establish the content of oligosaccharide in the ingredient powder (Table 1). The analysis of multiplicity and chemical shift of the ${ }^{1} \mathrm{H}-\mathrm{NMR}$ and the ${ }^{13} \mathrm{C}-\mathrm{NMR}$ signals were in accordance with the structures of LNnT and $2^{\prime}$-FL and checked by 2-dimensinal heteronuclear single quantum coherence (HSQC) and heteronuclear multiple bond correlation (HMBC) experiments. In most cases the ingredient powders contained more than $90 \%$ of the oligosaccharide, and the remaining mass of powder was predominantly moisture (Table 1). The data obtained from qNMR enabled us to use the ingredients as quantitative standards during validation studies. Aliquots of some batches were used as analytical standards, while the remaining material was used as material for laboratory spiking experiments and for pilot plant trials. 
Table 1. Human milk oligosaccharides (HMO) content of different batches of HMO.

\begin{tabular}{lccc}
\hline HMO & Batch & HMO Content of Ingredient (\%) & Moisture Content of Ingredient (\%) \\
\hline LNnT & A & 91.9 & n.d. \\
LNnT & B & 90.6 & 9.0 \\
LNnT & C & 94.5 & 5.1 \\
LNnT & D & 95.6 & 4.8 \\
LNnT & E & 95.5 & 4.7 \\
LNnT & F & 95.5 & 4.8 \\
LNnT & G & 90.8 & 9.1 \\
$2^{\prime}-$ FL & A & 89.3 & n.d. \\
$2^{\prime}-$ FL & B & 99.3 & n.d. \\
$2^{\prime}-$ FL & C & 99.1 & 0.5 \\
$2^{\prime}-$ FL & D & 97.0 & 2.6 \\
$2^{\prime}-$ FL & E & 96.1 & 3.2 \\
$2^{\prime}-$ FL & F & 96.0 & 2.9 \\
$2^{\prime}-$ FL & G & 95.7 & 2.4 \\
$2^{\prime}-$ FL & H & 89.7 & 3.5 \\
\hline
\end{tabular}

2'-FL = 2'-fucosyllactose; LNnT = Lacto-N-neotetraose; n.d. = not determined.

\subsection{Determination of $2^{\prime}-F L$ and $L N n T$ by HPAEC-PAD}

2'-FL and LNnT were well separated from each other and from lactose by HPAEC-PAD (Figure 1). To determine the calibration model, solutions of LNnT and $2^{\prime}-\mathrm{FL}$ were prepared at nine different concentrations (from 29 to $118 \mathrm{mg} / \mathrm{L}$ for LNnT and from 60 to $222 \mathrm{mg} / \mathrm{L}$ for $2^{\prime}-\mathrm{FL}$ ) in triplicate, and all the solutions were injected on the HPAEC-PAD system. The detector response was found to be linear for both oligosaccharides. For LNnT the slope of the curve was 0.97 and the $Y$ intercept was at 0.688 , with an $\mathrm{r}^{2}$ of 0.999 . For $2^{\prime}$-FL the slope of the curve was 0.46 and the Y-intercept was -1.91 with an $\mathrm{r}^{2}$ of 0.998 .

The limit of detection (LoD) and limit of quantification (LoQ) were determined by analyzing oligosaccharide standards at five different concentrations in triplicate between 1 and $20 \mu \mathrm{g} / \mathrm{mL}$. Plots of signal to noise $(\mathrm{S} / \mathrm{N})$ ratio against concentration were prepared for $2^{\prime}-\mathrm{FL}$ and $\mathrm{LNnT}$. LoDs at S/N ratio of 3 were determined to be $4.33 \mu \mathrm{g} / \mathrm{mL}$ for $2^{\prime}-\mathrm{FL}$ and $0.48 \mu \mathrm{g} / \mathrm{mL}$ for LNnT. LoQs at S/N ratio of 10 were determined to be $12.8 \mu \mathrm{g} / \mathrm{mL}$ for $2^{\prime}-\mathrm{FL}$ and $1.51 \mu \mathrm{g} / \mathrm{mL}$ for LNnT. Starting with a sample amount of $2 \mathrm{~g}$ dissolved in $100 \mathrm{~mL}$, the LoD and LoQ of $2^{\prime}-\mathrm{FL}$ in a product were estimated to be $0.02 \mathrm{~g} / 100 \mathrm{~g}$ and $0.06 \mathrm{~g} / 100 \mathrm{~g}$ respectively, and the LoD and LoQ of LNnT in a product were estimated to be $0.003 \mathrm{~g} / 100 \mathrm{~g}$ and $0.008 \mathrm{~g} / 100 \mathrm{~g}$ respectively.

The trueness and precision of the HPAEC-PAD method were assessed by analyzing two different infant formula samples spiked with the oligosaccharides at three different levels (Table 2). Spike-recoveries were in the range 94 to $99 \%$ for 2'-FL and 98 to $111 \%$ for LNnT. Repeatabilities were in the range of 0.47 to $5.2 \%$ for $2^{\prime}-\mathrm{FL}$, and 1.1 to $5.6 \%$ for $\mathrm{LNnT}$ and intermediate reproducibilities were in the range 2.2 to $5.7 \%$ for $2^{\prime}$-FL and 2.1 to $7.9 \%$ for LNnT. 


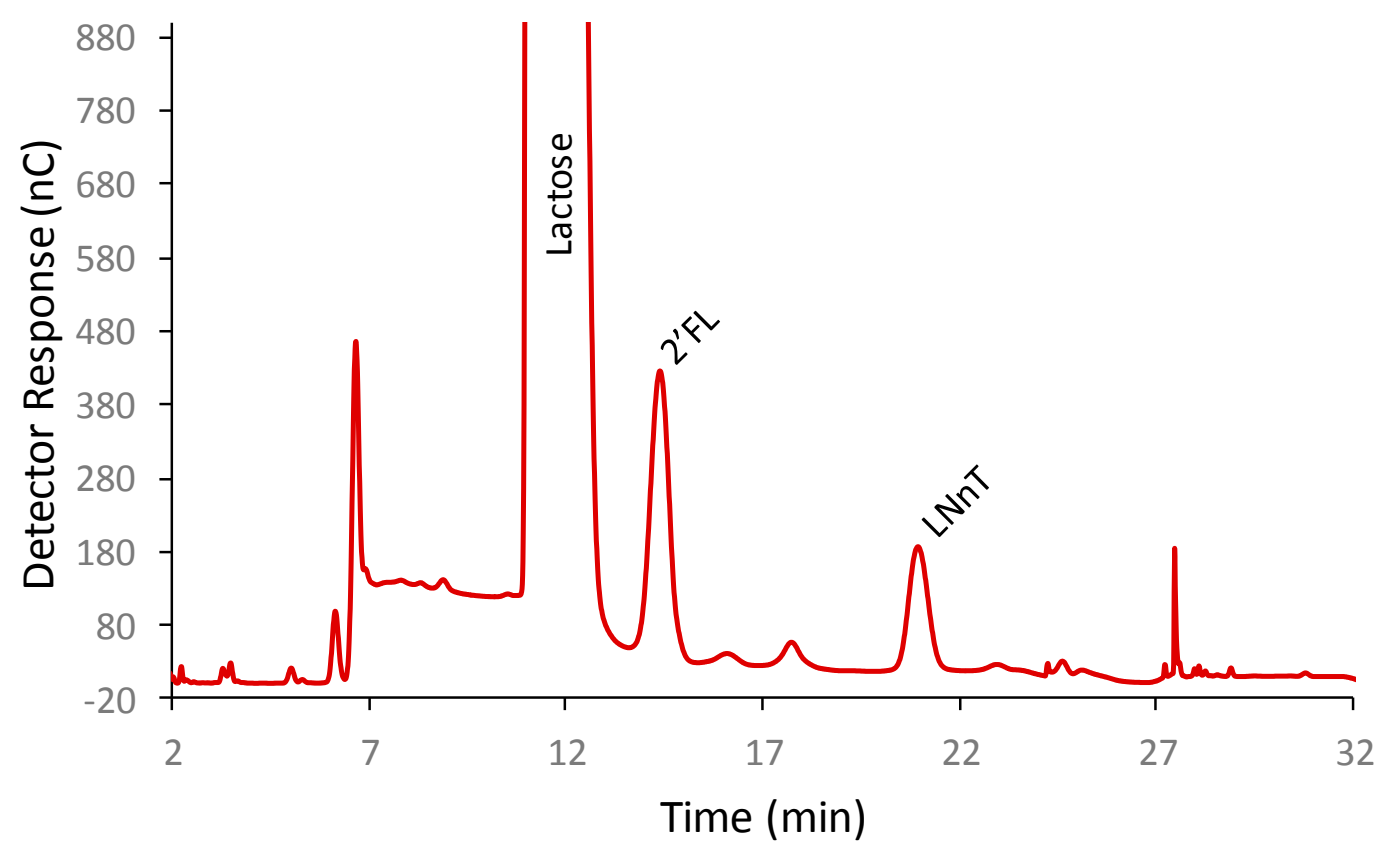

Figure 1. Separation of $2^{\prime}$-fucosyllactose $\left(2^{\prime}\right.$-FL) and Lacto-N-neotetraose (LNnT) from each other and from lactose in infant formula by high performance anion exchange chromatography with pulsed amperometric detection (HPAEC-PAD). 
Table 2. Performance of the method determined during validation.

\begin{tabular}{|c|c|c|c|c|c|c|c|c|c|c|c|}
\hline \multirow{2}{*}{ Method } & \multirow{2}{*}{ Matrix } & \multicolumn{2}{|c|}{ Spike (g/100 g) } & \multicolumn{2}{|c|}{ Measured (g/100 g) } & \multicolumn{2}{|c|}{ Recovery (\%) } & \multicolumn{2}{|c|}{ RSD(r) (\%) } & \multicolumn{2}{|c|}{ RSD (iR) (\%) } \\
\hline & & $2^{\prime}-\mathrm{FL}$ & LNnT & $2^{\prime}-\mathrm{FL}$ & LNnT & $2^{\prime}-\mathrm{FL}$ & LNnT & $2^{\prime}-\mathrm{FL}$ & LNnT & $2^{\prime}-\mathrm{FL}$ & LNnT \\
\hline \multirow{3}{*}{ HPAEC-PAD } & \multirow{3}{*}{ IF powder with intact protein } & 0.590 & 0.204 & 0.583 & 0.226 & 98.9 & 111 & 2.7 & 3.5 & 4.2 & 7.2 \\
\hline & & 0.751 & 0.357 & 0.735 & 0.367 & 97.8 & 103 & 1.7 & 1.1 & 2.2 & 2.1 \\
\hline & & 0.921 & 0.510 & 0.886 & 0.501 & 96.2 & 98.1 & 0.47 & 1.2 & 2.2 & 3.2 \\
\hline \multirow{3}{*}{ HPAEC-PAD } & \multirow{3}{*}{ IF powder with partially hydrolyzed protein } & 0.590 & 0.204 & 0.573 & 0.222 & 97.0 & 109 & 5.2 & 5.6 & 4.3 & 7.9 \\
\hline & & 0.751 & 0.357 & 0.710 & 0.363 & 94.6 & 102 & 4.8 & 4.9 & 5.7 & 5.6 \\
\hline & & 0.921 & 0.510 & 0.870 & 0.499 & 94.4 & 97.9 & 1.6 & 1.7 & 2.4 & 3.7 \\
\hline \multirow{3}{*}{ HILIC-FLD } & \multirow{3}{*}{ IF powder with intact protein } & 0.571 & 0.194 & 0.557 & 0.191 & 97.5 & 98.8 & 1.2 & 2.1 & 2.7 & 2.7 \\
\hline & & 0.723 & 0.338 & 0.702 & 0.331 & 97.1 & 97.9 & 1.7 & 1.9 & 3.3 & 3.2 \\
\hline & & 0.880 & 0.484 & 0.857 & 0.473 & 97.4 & 97.9 & 1.9 & 2.3 & 2.8 & 3.1 \\
\hline HILIC-FLD & IF powder with partially hydrolyzed protein & 0.822 & 0.425 & 0.805 & 0.397 & 97.9 & 93.5 & 1.7 & 1.2 & 2.7 & 4.4 \\
\hline \multirow{3}{*}{ HILIC-FLD } & \multirow{3}{*}{ IF powder with intact protein and FOS } & 0.573 & 0.193 & 0.570 & 0.188 & 99.6 & 97.4 & 1.8 & 2.4 & 2.7 & 4.7 \\
\hline & & 0.722 & 0.384 & 0.710 & 0.379 & 98.3 & 98.5 & 1.6 & 1.5 & 3.4 & 2.1 \\
\hline & & 0.874 & 0.582 & 0.855 & 0.579 & 97.9 & 99.5 & 1.2 & 1.5 & 2.6 & 2.5 \\
\hline \multirow{3}{*}{ HILIC-FLD } & \multirow{3}{*}{ FUF powder with intact protein and FOS } & 0.575 & 0.191 & 0.557 & 0.189 & 96.8 & 99.1 & 2.1 & 1.8 & 7.4 & 4.9 \\
\hline & & 0.722 & 0.387 & 0.695 & 0.390 & 96.3 & 101 & 2.0 & 1.6 & 4.5 & 2.6 \\
\hline & & 0.877 & 0.567 & 0.824 & 0.576 & 93.9 & 102 & 2.2 & 1.4 & 4.2 & 2.3 \\
\hline \multirow{3}{*}{ HILIC-FLD } & \multirow{3}{*}{ IF RTF with intact protein and FOS } & $0.541^{\mathrm{a}}$ & $0.222^{a}$ & $0.548^{\mathrm{a}}$ & $0.224^{\mathrm{a}}$ & 101 & 101 & 1.5 & 2.3 & 2.6 & 3.5 \\
\hline & & $1.06^{\mathrm{a}}$ & $0.546^{\mathrm{a}}$ & $1.06^{\mathrm{a}}$ & $0.568^{\mathrm{a}}$ & 100 & 104 & 1.8 & 2.0 & 2.0 & 2.5 \\
\hline & & $4.17^{\mathrm{a}}$ & $1.05^{\mathrm{a}}$ & $4.19^{\mathrm{a}}$ & $1.09^{\mathrm{a}}$ & 101 & 104 & 1.2 & 2.6 & 2.2 & 4.8 \\
\hline HILIC-FLD & $\begin{array}{l}\text { IF powder with intact protein and FOS } \\
\text { (pilot production) }\end{array}$ & $\mathrm{n} / \mathrm{a}$ & $\mathrm{n} / \mathrm{a}$ & 0.785 & 0.390 & $\mathrm{n} / \mathrm{a}$ & $\mathrm{n} / \mathrm{a}$ & 1.5 & 2.2 & 3.3 & 5.0 \\
\hline HILIC-FLD & $\begin{array}{l}\text { FUF powder with intact protein and FOS } \\
\text { (pilot production) }\end{array}$ & $\mathrm{n} / \mathrm{a}$ & $\mathrm{n} / \mathrm{a}$ & 0.600 & 0.304 & $\mathrm{n} / \mathrm{a}$ & $\mathrm{n} / \mathrm{a}$ & 1.3 & 1.8 & 5.0 & 3.1 \\
\hline
\end{tabular}

${ }^{a}$ Values in $\mathrm{g} / \mathrm{L}$. Abbreviations: FOS = fructooligosaccharides, FUF = follow-up-formula, IF = Infant formula, RTF = ready to feed. 


\subsection{Determination of 2'-FL and LNnT by HILIC-FLD}

2'-FL and LNnT were well separated from each other, from lactose, and from the laminaritriose internal standard using HILIC-FLD (Figure 2). To investigate the calibration model we split the concentration range for both $\mathrm{HMO}$ in to two ranges, a low range (covering $2^{\prime}$-FL concentrations from 5 to $293 \mathrm{mg} / \mathrm{L}$ and LNnT concentrations from 7 to $426 \mathrm{mg} / \mathrm{L}$ ) and a high range (covering 2'-FL concentrations from 244 to $2931 \mathrm{mg} / \mathrm{L}$ and LNnT concentrations from 355 to $4255 \mathrm{mg} / \mathrm{L}$ ). For the low range, oligosaccharide solutions were prepared at nine different concentrations in triplicate. For the high range, oligosaccharide solutions were prepared at seven different concentrations in triplicate. In all cases a linear model described the relationship between detector response and concentration. For 2'-FL at the low range the slope was 0.008 and Y-intercept -0.001 , with an $r^{2}$ of 0.998 . For 2'-FL at the high range the slope was 0.008 and Y-intercept -0.150 , with an $\mathrm{r}^{2}$ of 0.994 . For LNnT at the low range the slope was 0.005 and Y-intercept -0.016 , with an $\mathrm{r}^{2}$ of 0.997 . For LNnT at the high range the slope was 0.005 and Y-intercept -0.188 , with an $\mathrm{r}^{2}$ of 0.994 .

LoD and LoQ were determined by analyzing four different formula powder products and one ready-to-feed liquid product without the addition of $\mathrm{HMO}$, and determining the chromatographic baseline noise at the retention time of $2^{\prime}-\mathrm{FL}$ and LNnT. Amounts of HMO required to generate a signal 10 times higher than the baseline noise were assigned as LoQ and amounts of HMO required to generate a signal 3 times higher than the baseline noise were assigned as $\mathrm{LoD}$ (for powder products the product with the highest values was assigned as the general LoD or LoQ). For 2'-FL in powdered products a small interfering peak was present close to the expected retention time of $2^{\prime}$-FL. In this case the peak area of the interfering peak was used as the baseline noise, and concentrations of $2^{\prime}$-FL giving rise to a peak area of 10 times or 3 times that of the interference peak were assigned as LOQ and LoD, respectively.

For powdered products, the LoDs of $2^{\prime}$-FL and LNnT were estimated to be $0.11 \mathrm{~g} / 100 \mathrm{~g}$ and $0.07 \mathrm{~g} / 100 \mathrm{~g}$, respectively, and for liquid products the LoD for $2^{\prime}$-FL and LNnT were estimated to be 0.01 and $0.03 \mathrm{~g} / \mathrm{L}$, respectively. For powdered products, the LoQs of 2'-FL and LNnT were estimated to be $0.37 \mathrm{~g} / 100 \mathrm{~g}$ and $0.13 \mathrm{~g} / 100 \mathrm{~g}$, respectively, and for liquid products, the LoQs for 2'-FL and LNnT were estimated to be $0.04 \mathrm{~g} / \mathrm{L}$ and $0.11 \mathrm{~g} / \mathrm{L}$, respectively.

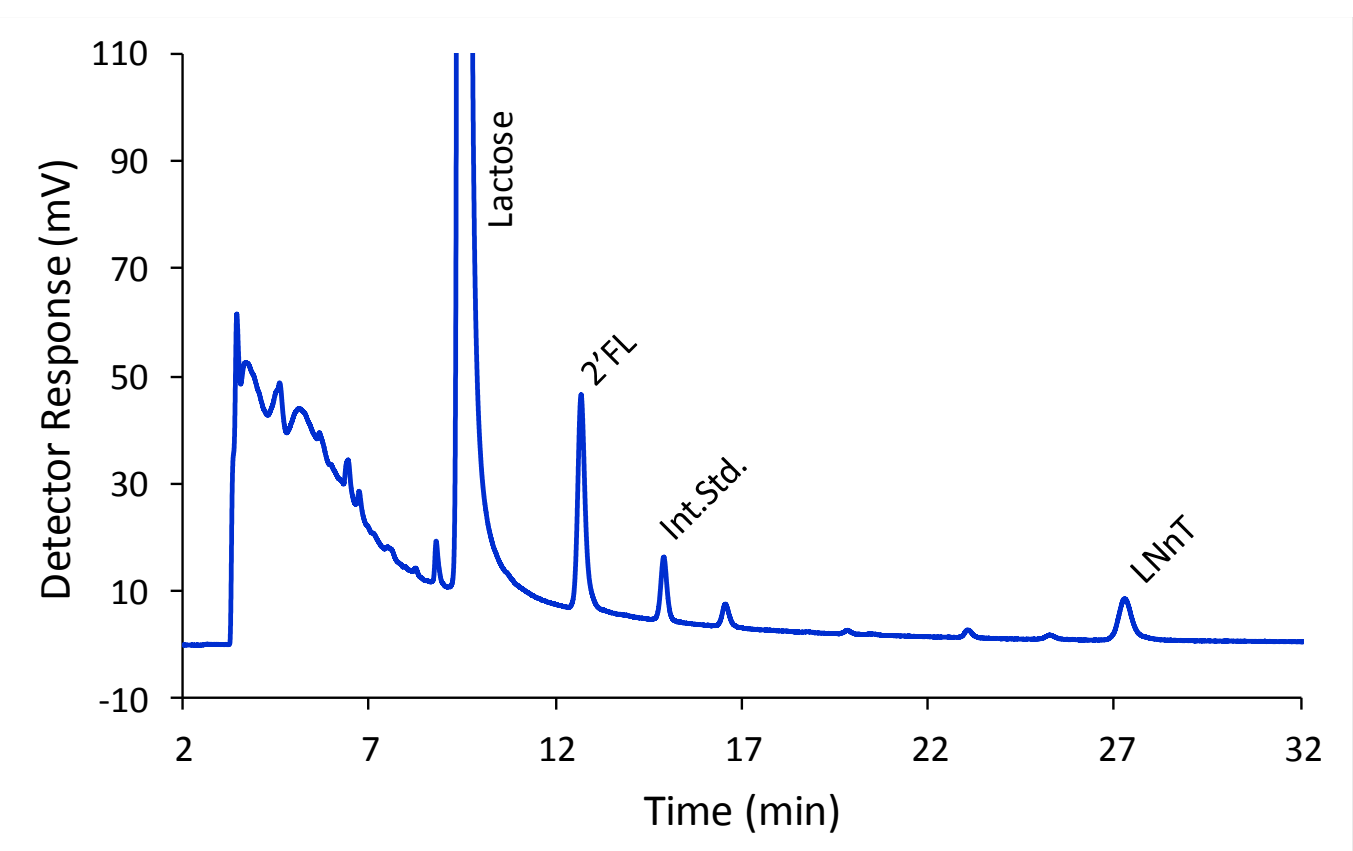

Figure 2. Separation of $2^{\prime}$-fucosyllactose $\left(2^{\prime}-\mathrm{FL}\right)$ and Lacto-N-neotetraose (LNnT) from each other and from lactose in infant formula by HILIC-FLD, using laminaritriose as an internal standard (Int. Std.). 
Trueness and precision of the method were assessed by analyzing four different infant formula powders spiked with different levels of 2'-FL and LNnT and by analyzing a ready to feed (RTF) formula spiked at three different levels with $2^{\prime}$-FL and LNnT (Table 2). Spike-recoveries were in the range 94 to $101 \%$ for $2^{\prime}$-FL and 94 to $104 \%$ for LNnT. Repeatabilities were in the range of 1.2 to $2.2 \%$ for $2^{\prime}$-FL, and 1.2 to $2.6 \%$ for LNnT and intermediate reproducibilities were in the range 2.0 to $7.4 \%$ for $2^{\prime}$-FL and 2.1 to $4.9 \%$ for LNnT, which are similar to the performance of the HPAEC-PAD method.

\subsection{Determination of $2^{\prime}-F L$ and LNnT in Pilot Plant and Commercial Samples}

Ten different formulae produced on pilot or industrial plants were analyzed using both approaches (Table 3). Surprisingly, for eight of the samples the HPAEC-PAD method returned results which were lower than those from the HILIC-FLD methods, and were also lower than the target concentrations where known. However for two of the samples (Formulas (5) and (6) in Table 3), the HPAEC-PAD method returned results similar to or higher than those from the HILIC-FLD.

Table 3. Determination of HMO in pilot plant or commercial formulae.

\begin{tabular}{|c|c|c|c|c|c|c|}
\hline \multirow{2}{*}{ Sample } & \multicolumn{3}{|c|}{$2^{\prime}-\mathrm{FL}(\mathrm{g} / 100 \mathrm{~g})$} & \multicolumn{3}{|c|}{ LNnT (g/100 g) } \\
\hline & Target & HILIC & HPAEC & Target & HILIC & HPAEC \\
\hline Formula (1): Intact Protein & 0.800 & $\mathrm{n} / \mathrm{a}^{(\mathrm{a})}$ & 0.625 & 0.400 & $0.452^{\text {(a) }}$ & 0.329 \\
\hline Formula (2): Intact protein & 0.800 & $\mathrm{n} / \mathrm{a}^{(\mathrm{a})}$ & 0.611 & 0.400 & $0.437^{(a)}$ & 0.331 \\
\hline Formula (3): Intact protein & 0.800 & $\mathrm{n} / \mathrm{a}^{(\mathrm{a})}$ & 0.630 & 0.400 & $0.379^{(a)}$ & 0.286 \\
\hline Formula (4): Intact protein & 0.800 & $\mathrm{n} / \mathrm{a}^{(\mathrm{a})}$ & 0.637 & 0.400 & $0.394^{(\mathrm{a})}$ & 0.288 \\
\hline Formula (5): Intact Protein & 0.800 & 0.822 & 0.904 & 0.400 & 0.402 & 0.410 \\
\hline Formula (6): Intact Protein & 0.800 & 0.824 & 0.914 & 0.400 & 0.416 & 0.412 \\
\hline Formula (7): Partially hydrolyzed protein & 0.190 & 0.173 & 0.141 & 0 & $\mathrm{nd}^{(\mathrm{b})}$ & nd ${ }^{(b)}$ \\
\hline Formula (8): Intact protein & Unk. (c) & 0.172 & 0.147 & 0 & nd ${ }^{(b)}$ & nd ${ }^{(b)}$ \\
\hline Formula (9): Intact protein & Unk. (c) & 0.170 & 0.123 & 0 & nd ${ }^{(b)}$ & nd ${ }^{(b)}$ \\
\hline Formula (10): Partially hydrolyzed protein & Unk. (c) & 0.708 & 0.664 & Unk. & 0.342 & 0.294 \\
\hline
\end{tabular}

(a) Determined using original GOS method [20] in which 2'-FL co-eluted with the internal standard, thus 2'-FL cannot be determined \& LNnT was determined using only an external standard. $\mathrm{n} / \mathrm{a}=$ not applicable. ${ }^{(\mathrm{b})}$ nd $=$ not detected $^{(\mathrm{c})}$ Unk. $=$ Target concentration is unknown.

\section{Discussion}

When we started developing methods for the analysis of $\mathrm{HMO}$, we used oligosaccharide standards purchased off the shelf from laboratory chemical suppliers. However, we rapidly ran into problems achieving oligosaccharide recoveries well in excess of 100\%. Troubleshooting exercises suggested that the purity of the standards was significantly less than the purity of the bulk material produced as ingredients. We therefore decided to use the $2^{\prime}$-FL and LNnT ingredients as our standards, but required a way to confirm the purity of the material. This was achieved using qNMR. Good quality HMO standards with qNMR data are not readily available from laboratory chemical vendors, but they can be obtained from some suppliers if specifically requested. Acquiring such standards remains something of a challenge. However, as more HMO become available as ingredients it is likely that good quality quantitative standards will become more readily obtainable.

We initially developed the method for $2^{\prime}$-FL and LNnT analysis on HPAEC-PAD, since it is a well-established technique for the analysis of oligosaccharides, and sample preparation is normally very simple. Validation of the HPAEC-PAD method was performed by dry mixing the HMO in to commercially available formula, and analyzing on six different days in duplicate. Method trueness and precision were both acceptable (Table 2). When we received samples from a pilot plant for analysis the results obtained by HPAEC-PAD were lower than expected from the addition rate (Table 3; formulas (1)-(4)). Investigations at the pilot plant confirmed that the correct addition rates had been applied, and it was not expected that there would be significant degradation of HMO under the processing conditions used. As part of the investigations in the analytical lab, we tried to analyze the samples 
using an alternative method. Since we were running galactooligosaccharide analysis at the time using HILIC-FLD [20], we decided to apply that method to HMO. We used the same internal standard (laminaritriose), sample preparation protocol, and LC conditions for the HMO analysis as we used for GOS analysis. Unfortunately, under those conditions, the 2'-FL co-eluted with the internal standard, thus it was not possible to quantify $2^{\prime}-\mathrm{FL}$, and the LNnT had to be quantified using an external calibration curve. Nevertheless, the measured LNnT concentrations were close to the expected content of $0.40 \mathrm{~g} / 100 \mathrm{~g}$ (Table 3. Formulas (1)-(4)). This led us to abandon the HPAEC-PAD method, and start development on the HILIC-FLD method.

The HILIC-FLD method was adapted from the GOS method by changing from HLPC to UHPLC, and adapting the column temperature and gradient conditions to avoid co-elution of $2^{\prime}$-FL with the internal standard. The HILIC-FLD method was also validated by dry-mixing HMO in to existing formula products. The validation data indicated that the HILIC-FLD method performed at least as well as the HPAEC-PAD method on spiked samples (Table 2). Two pilot plant samples were also included in the validation to assess precision (Table 2) in samples which were expected to be more homogenous than the dry-mixed lab samples. The RSD(r) and RSD(iR) determined on the pilot plant samples were similar to the samples from spiking experiments at $5 \%$ or less.

A selection of commercial and pilot plant samples was analyzed by both the HILIC-FLD method and the HPAEC-PAD method (Table 3). In general, the results obtained by HILIC-FLD tended to be higher than those obtained by HPAEC-PAD. We hypothesize that there are some interactions between the analytes and the matrix which may be initiated during the industrial production process. That interaction is not completely disrupted when samples are simply extracted in warm water, and thus the analysis by HPAEC-PAD seems to underestimate $2^{\prime}$-FL and LNnT. The more aggressive conditions used during the oligosaccharide labeling procedure (using low $\mathrm{pH}$ and DMSO as solvent) seem to disrupt those interactions, and thus the results from the HILIC-FLD method are more in line with expectations. However, as can be seen from data on commercial and pilot plant samples (Table 3), the HPAEC-PAD method does not always underestimate the content of the HMO (see formula (5) and (6) in Table 3). Thus the hypothesis is not universal for all products, and is probably dependent on the product recipe and specific processing conditions.

\section{Materials and Methods}

\subsection{Samples}

Infant formula samples were obtained from Nestlé Product Technology Centre (Konolfingen, Switzerland), from Wyeth Nutrition (Askeaton, Ireland), or from Nestlé Nutrition (Vevey, Switzerland).

\subsection{Chemicals}

All water was deionized (18 M $\Omega$ ) produced by a Milli-Q system (Merck Millipore, Darmstadt, Germany), sodium hydroxide solution (50\%) was from JT Baker (Deventer, The Netherlands), 2'-FL and LNnT were obtained from Glycom (Hørsholm, Denmark), all other chemicals were of analytical grade or higher and sourced from Sigma-Aldrich/Merck, Darmstadt, Germany.

\subsection{Determination of 2'-FL and LNnT Purity by Quantitative NMR}

NMR measurements were performed on Bruker Avance III 600 spectrometer (Bruker, Karlsruhe, Germany) equipped with a QNP cryo probe head and a Bruker automatic sample changer (B-ACS 120) or on a Bruker Avance III HD 500 spectrometer equipped with a BBO prodigy cryo probe and a Bruker automatic sample changer (B-ACS 120).

For quantification of oligosaccharides, appropriate amounts of the oligosaccharide and of nicotinic acid amide (NSA) internal standard were weighed exactly, dissolved in $1 \mathrm{ml}$ D2O, and measured. Integrated signals of the oligosaccharide and of the internal standard were used for calculation. The ratio of integrals per atom corresponds to the molar ratio of the compared substances. 
The amount of water in the samples was determined by ${ }^{1} \mathrm{H}-\mathrm{NMR}$ in DMSO- $d_{6}$. The water signal appears as a separated signal in DMSO and no chemical exchange was observed with the $\mathrm{OH}$ protons of the oligosaccharides. The ratio of the water signal representing 2 protons per molecule and a suitable signal of the oligosaccharide (e.g., a hydroxyl resonance with 1 proton per molecule) were used to calculate the molar ratio, after correcting for residual water present in the DMSO solvent. Using the different molecular weights of water and the corresponding oligosaccharide it was possible to calculate the mass balance.

\subsection{Determination of $2^{\prime}-F L \mathcal{E} L N n T$ in Formula by HPAEC-PAD}

The infant formula sample $(2 \mathrm{~g})$ was mixed with water $(70 \mathrm{~mL})$ and heated at $70{ }^{\circ} \mathrm{C}$ for $25 \mathrm{~min}$ with constant stirring. The sample was then clarified by the addition of Carrez I solution $(1 \mathrm{~mL})$ followed by the addition of Carrez II solution $(1 \mathrm{~mL})$ and sodium hydroxide $(1 \mathrm{~mol} / \mathrm{L} ; 0.7 \mathrm{~mL})$ and made up to $100 \mathrm{~mL}$ with water in a volumetric flask. A portion of the clarified solution was filtered through a $0.22 \mu \mathrm{m}$ nylon membrane filter in to a LC autosampler vial.

The oligosaccharides were separated on a CarobPac PA1 column $(4 \times 250 \mathrm{~mm})$ using an ICS 3000 high performance anion exchange chromatography system composed of an autosampler, quaternary gradient pump, column oven and pulsed electrochemical detector with a gold working electrode and silver/silver chloride reference electrode (all from Thermo Fisher Scientific, Waltham, MA, USA). Separation was achieved using the gradient described in Table 4 at a flow of $1 \mathrm{~mL} / \mathrm{min}$ and column temperature of $30^{\circ} \mathrm{C}$. Injection volume was $10 \mu \mathrm{L}$.

Table 4. Gradient for determination of 2'-FL and LNnT by HPAEC-PAD.

\begin{tabular}{ccc}
\hline Time $(\mathbf{m i n})$ & Sodium Hydroxide $(\mathbf{m M})$ & Sodium \\
\hline 0 & 50 & 0 \\
2.0 & 50 & 0 \\
18.0 & 110 & 0 \\
18.1 & 110 & 12.5 \\
25.0 & 110 & 12.5 \\
25.1 & 150 & 500 \\
30.0 & 150 & 500 \\
30.1 & 300 & 0 \\
35.0 & 300 & 0 \\
35.1 & 50 & 0 \\
40.0 & 50 & 0 \\
\hline
\end{tabular}

\subsection{Determination of $2^{\prime}-F L$ and LNnT in Formula by HILIC-FLD}

The infant formula sample ( $1 \mathrm{~g}$ for powder products, $14 \mathrm{~mL}$ for liquid products) was mixed with $70 \mathrm{~mL}$ of water and heated at $70^{\circ} \mathrm{C}$ for $25 \mathrm{~min}$ with constant stirring. The cooled solution was diluted to $100 \mathrm{~mL}$ with deionized water in a volumetric flask. An aliquot $(500 \mu \mathrm{L})$ of this solution was transferred to a plastic microtube and laminaritriose solution $(0.3 \mu \mathrm{mol} / \mathrm{mL}, 200 \mu \mathrm{L})$ was added. An aliquot $(20 \mu \mathrm{L})$ of this solution was transferred to another microtube, and labeling reagent (2-aminobenzamide $(0.35 \mathrm{~mol} / \mathrm{L})+$ sodium cyanoborohydride $(1.0 \mathrm{~mol} / \mathrm{L})$ in DMSO containing 30\% acetic acid; $200 \mu \mathrm{L})$ was added. After mixing the sealed tube was placed in a water bath at $65^{\circ} \mathrm{C}$ for $2 \mathrm{~h}$. After cooling, the mixture was diluted with acetonitrile/water $(7 / 3 ; 1500 \mu \mathrm{L})$, particles were removed by centrifugation $(10,000 \times g ; 5 \mathrm{~min})$, and a portion of the liquid was transferred to a liquid chromatography (LC) autosampler vial. The labeled oligosaccharides were separated by HILIC on a BEH Glycan column $(1.7 \mu \mathrm{m}, 2.1 \times 150 \mathrm{~mm})$ preceded by a BEH amide guard column $(1.7 \mu \mathrm{m}, 2.1 \times 50 \mathrm{~mm})$ both from Waters Corporation (Milford, CT, USA). A 2-way 10-port valve was connected between the guard and analytical columns, this was used to direct the eluent from the guard column to waste while excess labeling reagents were eluted, the valve was then switched to direct the flow through the analytical column for separation of the oligosaccharides. The analytical column was held at $60{ }^{\circ} \mathrm{C}$, and the 
guard column was kept at room temperature $\left(20-25^{\circ} \mathrm{C}\right)$. Separation was achieved using a gradient of ammonium formate (100 mol/L; pH 4.4) and acetonitrile (Table 5). The eluted oligosaccharides were detected by fluorescence using an excitation wavelength of $330 \mathrm{~nm}$ and an emission wavelength of $420 \mathrm{~nm}$. The LC system was an Ultimate $3000 \mathrm{RS}$, with an upper pressure limit of 1000 Bar, connected to a RF2000 fluorimeter (all from Thermo Fisher Scientific), the injection volume was $1 \mu \mathrm{L}$.

Table 5. Gradient for separation of HMO by hydrophilic interaction liquid chromatography with fluorescence detection (HILIC-FLD).

\begin{tabular}{ccccc}
\hline Time (min) & Flow $(\mathbf{m L} / \mathbf{m i n})$ & $\mathbf{\% A}$ & $\mathbf{\% B}$ & Valve \\
\hline 0 & 0.6 & 98 & 2 & waste \\
2.5 & 0.6 & 98 & 2 & analyse \\
3.0 & 0.6 & 88 & 12 & analyse \\
10.0 & 0.6 & 88 & 12 & analyse \\
20 & 0.6 & 83 & 17 & analyse \\
33.0 & 0.6 & 83 & 17 & analyse \\
34.0 & 0.45 & 30 & 70 & analyse \\
37.0 & 0.45 & 30 & 70 & analyse \\
39.0 & 0.45 & 90 & 10 & analyse \\
45.0 & 0.6 & 90 & 10 & analyse \\
45.1 & 0.6 & 98 & 2 & waste \\
46 & 0.6 & 98 & 2 & waste \\
\hline
\end{tabular}

Eluent $\mathrm{A}=$ acetonitrile; eluent $\mathrm{B}=$ ammonium formate $(100 \mathrm{mmol} / \mathrm{L}, \mathrm{pH} 4.4)$.

\subsection{Method Validation}

To check the calibration model of both methods, three independent calibration curves were prepared, each containing at least nine different HMO concentrations. For both analytes, a linear calibration model was applied. The fit of the calibration model was checked by determination of $\mathrm{r}^{2}$ and using plots of the residuals.

For the HPAEC-PAD method, limits of detection (LoD) and quantification (LoQ) were determined by analyzing solutions of the $\mathrm{HMO}$ at low concentrations, and measuring the signal to noise (S/N) ratio. Concentrations resulting in a $\mathrm{S} / \mathrm{N}$ ratio of 3 were designated as LoD and those resulting in a $\mathrm{S} / \mathrm{N}$ ratio of 10 were designated as LoQ. From those concentrations and the procedure for sample preparation is was possible to back-calculate the equivalent LoD and LoQ in a formula powder.

For the HILIC-FLD method, a similar approach as for HPAEC-PAD was followed, however multiple analyses of blank formula were used to estimate the noise level for the chromatogram. Concentrations of $\mathrm{HMO}$ resulting in a $\mathrm{S} / \mathrm{N}$ ratio of 3 were designated as LoD and those resulting in a $\mathrm{S} / \mathrm{N}$ ratio of 10 were designated as provisional LoQ. These LoDs and LoQs were then compared to the lowest point on the calibration curve, and the highest value (from all the $S / N$ experiments or from the lowest point on the standard curve) was assigned as the definitive LoD or LoQ.

To check the accuracy and precision of the HPAEC-PAD method, two infant formula samples (a whey-predominant formula with intact protein, and a whey-predominant formula with partially hydrolyzed proteins) which did not contain any nondigestible oligosaccharides were spiked (dry blended) with LNnT and 2'-FL, each at three different concentrations and the samples analyzed in duplicate on six different days.

To check the accuracy and precision of the HILIC-FLD method, one infant formula powder (a whey-predominant formula with partially hydrolyzed protein) which did not contain any nondigestible oligosaccharides was spiked with LNnT and 2'-FL at one level. One infant formula powder (a whey-predominant formula with intact protein) which did not contain any nondigestible oligosaccharides was spiked with LNnT and 2'-FL at three different levels. One infant formula powder, and one follow-up formula powder (both whey-predominant formula with intact protein) containing fructooligosaccharides (FOS) were spiked with LNnT and 2'-FL at three different levels. One ready-to-feed formula (whey-predominant formula with intact protein) containing FOS was 
spiked with LNnT and 2'-FL at three different levels. Spikes in powder products were performed by dry blending in the lab. All samples were analyzed in duplicate on six different days.

Method accuracy was determined by comparing the HMO levels measured with the amount of HMO that had been spiked in the formula. Method precision was determined by estimating the relative standard deviation of the results determined under repeatability $(\operatorname{RSD}(\mathrm{r}))$ or intermediate reproducibility $(\mathrm{RSD}(\mathrm{iR}))$ conditions.

All data were processed using the in-house statistical program QStat.net using the equations that have been previously described [13].

\section{Conclusions}

$2^{\prime}$-FL and LNnT can be accurately determined in infant formula using HILIC-FLD after labeling the oligosaccharides with $2 \mathrm{AB}$. The labeling reaction probably disrupts interactions between the analytes and the matrix, which may be responsible for the lower results sometimes observed when HPAEC-PAD is used for the analysis of samples coming from pilot or industrial plants.

Author Contributions: Conceptualization, S.A. and B.C.; Methodology, S.A., B.D., J.M., D.C., and B.C.; Validation, J.M., D.C., and B.C. Formal Analysis, J.M., D.C., and B.D.; Investigation, B.D., J.M., D.C., and B.C.; Writing-Original Draft Preparation, S.A.; Writing-Review \& Editing, S.A., B.D., D.C., B.C., and J.M.; Supervision, S.A.; Visualization, S.A.

Funding: This research received no external funding.

Acknowledgments: The authors would like to thank Andrea Forray at the Nestle Product Technology Centre, Konolfingen, Switzerland for the production of formula samples containing HMO and the staff at Glycom, Hørsholm, Denmark for their support and production of HMO. We also thank Dominique Brassart, Nestlé Nutrition, Vevey, Switzerland and Norbert Sprenger, Nestlé Research Centre, Lausanne, Switzerland for their support and project management.

Conflicts of Interest: S.A., J.M., D.C., and B.C. were all employees of Nestec Ltd. when this work was performed; B.D. declares no conflict of interest.

\section{References}

1. Oliveira, D.L.; Wilbey, R.A.; Grandison, A.S.; Boseiro, L.B. Milk oligosaccharides: A review. Int. J. Dairy Tech. 2015, 68, 305-321. [CrossRef]

2. Bode, L. The functional biology of human milk oligosaccharides. Early Hum. Dev. 2015, 91, 619-622. [CrossRef] [PubMed]

3. He, Y.; Liu, S.; Kling, D.E.; Leone, S.; Lawlor, N.T.; Huang, Y.; Feinberg, S.B.; Hill, D.R.; Newburg, D.S. The human milk oligosaccharide 2 '-fucosyllactose modulates cd14 expression in human enterocytes, thereby attenuating LPS-induced inflammation. Gut 2016, 65, 33-46. [CrossRef] [PubMed]

4. Matsuki, T.; Yahagi, K.; Mori, H.; Matsumoto, H.; Hara, T.; Tajima, S.; Ogawa, E.; Kodama, H.; Yamamoto, K.; Yamada, T.; et al. A key genetic factor for fucosyllactose utilization affects infant gut microbiota development. Nat. Commun. 2016, 7, 11939. [CrossRef] [PubMed]

5. Smith-Brown, P.; Morrison, M.; Krause, L.; Davies, P.S. Mothers secretor status affects development of childrens microbiota composition and function: A pilot study. PLoS ONE 2016, 11, e0161211. [CrossRef] [PubMed]

6. Urashima, T.; Hirabayashi, J.; Sato, S.; Kobata, A. Human milk oligosaccharides as essential tools for basic and application studies on galectins. Trends Glycosci. Glyc. 2018, 30, SE51-SE56. [CrossRef]

7. Thurl, S.; Munzert, M.; Boehm, G.; Matthews, C.; Stahl, B. Systematic review of the concentrations of oligosaccharides in human milk. Nutr. Rev. 2017, 75, 920-933. [CrossRef] [PubMed]

8. Musumeci, M.; Simpore, J.; D’Agata, A.; Sotgiu, S.; Musumeci, S. Oligosaccharides in colostrum of Italian and Burkinabe women. J. Pediatr. Gastroenterol. Nutr. 2006, 43, 372-378. [CrossRef] [PubMed]

9. Morrow, A.L.; Ruiz-Palacios, G.M.; Altaye, M.; Jiang, X.; Guerrero, M.L.; Meinzen-Derr, J.K.; Farkas, T.; Chaturvedi, P.; Pickering, L.K.; Newburg, D.S. Human milk oligosaccharides are associated with protection against diarrhea in breast-fed infants. J. Pediatr. 2004, 145, 297-303. [CrossRef] [PubMed]

10. Newburg, D.S.; Ruiz-Palacios, G.M.; Morrow, A.L. Human milk glycans protect infants against enteric pathogens. Annu. Rev. Nutr. 2005, 25, 37-58. [CrossRef] [PubMed] 
11. Newburg, D.S.; Ruiz-Palacios, G.M.; Altaye, M.; Chaturvedi, P.; Meinzen-Derr, J.; Guerrero, M.D.; Morrow, A.L. Innate protection conferred by fucosylated oligosaccharides of human milk against diarrhea in breastfed infants. Glycobiology 2004, 14, 253-263. [CrossRef] [PubMed]

12. Nakhla, T.; Daotian, F.; Zopf, D.; Brodsky, N.L.; Hurt, H. Neutral oligosaccharide content of preterm human milk. Brit. J. Nutr. 1999, 82, 361-367. [CrossRef] [PubMed]

13. Galeotti, F.; Coppa, G.V.; Zampini, L.; Maccari, F.; Galeazzi, T.; Padella, L.; Santoro, L.; Gabrielli, O.; Volpi, N. Capillary electrophoresis separation of human milk neutral and acidic oligosaccharides derivatized with 2-aminoacridone. Electrophoresis 2014, 35, 811-818. [CrossRef] [PubMed]

14. Sprenger, G.A.; Baumgartner, F.; Albermann, C. Production of human milk oligosaccharides by enzymatic and whole-cell microbial biotransformations. J. Biotechnol. 2017, 258, 79-91. [CrossRef] [PubMed]

15. Marriage, B.J.; Buck, R.H.; Goehring, K.C.; Oliver, J.S.; Williams, J.A. Infants fed a lower calorie formula with 2'-FL show growth and 2'-FL uptake like breast-fed infants. J. Pediatr. Gastroenterol. Nutr. 2015, 61, 649-658. [CrossRef] [PubMed]

16. Goehring, K.C.; Marriage, B.J.; Oliver, J.S.; Wilder, J.A.; Barrett, E.G.; Buck, R.H. Similar to those who are breastfed, infants fed a formula containing $2^{\prime}$-fucosyllactose have lower inflammatory cytokines in a randomized controlled trial. J. Nutr. 2016, 146, 2559-2566. [CrossRef] [PubMed]

17. Puccio, G.; Alliet, P.; Cajozzo, C.; Janssens, E.; Corsello, G.; Sprenger, N.; Wernimont, S.; Egli, D.; Gosoniu, L.; Steenhout, P. Effects of infant formula with human milk oligosaccharides on growth and morbidity: A randomized multicenter trial. J. Pediatr. Gastroenterol. Nutr. 2017, 64, 624-631. [CrossRef] [PubMed]

18. Thurl, S.; Muller, W.B.; Sawatzki, G. Quantification of individual oligosaccharide compounds from human milk using high-pH anion-exchange chromatography. Anal. Biochem. 1996, 235, 202-206. [CrossRef] [PubMed]

19. Jantscher-Krenn, E.; Lauwaet, T.; Bliss, L.A.; Reed, S.L.; Gillin, F.D.; Bode, L. Human milk oligosaccharides reduce Entamoeba histolytica attachment and cytotoxicity in vitro. Br. J. Nutr. 2012, 108, 1839-1846. [CrossRef] [PubMed]

20. Austin, S.; Benet, T.; Michaud, J.; Cuany, D.; Rohfritsch, P. Determination of $\beta$-galactooligosaccharides by liquid chromatography. Int. J. Anal. Chem. 2014, 2014. [CrossRef] [PubMed]

Sample Availability: Samples of the HMO are available from the authors.

(C) 2018 by the authors. Licensee MDPI, Basel, Switzerland. This article is an open access article distributed under the terms and conditions of the Creative Commons Attribution (CC BY) license (http:// creativecommons.org/licenses/by/4.0/). 